Proceedings of SPIE's 1991 International Symposium on optical

Science and Engineering, 21-26 July, San Diego, CA.

conf. \# 1552 Short Wavelength Radiation Sources, paper \#25 CONF- $9107 / 15^{5}-85$

\title{
PRODUCTION OF X-RAYS BY THE INTERACTION OF CHARGED PARTICLE BEAMS WITH PERIODIC STRUCTURES AND CRYSTALLINE MATERIALS
}

\author{
D. W. Rule and R. B. Fiorito \\ Naval Surface Warfare Center \\ 10901 New Hampshire Avenue, Code R42 \\ Silver Spring, Maryland 20903-5000 \\ M. A. Piestrup and C. K. Gary \\ Adelphi Technologies Incorporated \\ 285 Hamilton Avenue - Suite 430 \\ Palo Alto, California 94301 \\ X. K. Maruyama \\ Naval Postgraduate School \\ Department of Physics \\ Monterey, California 93943
}

\begin{abstract}
We describe our recent experimental study of the production of $x-r a y s$ by an electron beam interacting with a crystal lattice, i.e. parametric $x-$ ray (PX) generation. In this radiation process, the virtual photon field associated with a relativistic electron traveling in a crystal is diffracted by the crystal lattice in the same way that real x-rays are diffracted by crystals. The radiation produced satisfies the Bragg condition associated with the diffraction of the virtual photons which are nearly parallel to the velocity of the electrons. This phenomenon is associated with a more general class of radiation production mechanisms which include transition radiation (TR), diffraction radiation (DR), and Smith-Purcell radiation. In each case, radiation is produced when the particle's fields are altered by interacting with a material whose dielectric constant varies along or near the particle's trajectory. The usual acceleration mechanism for the production of radiation is not involved in these phenomena. In the case of a crystal, the periodic electric susceptibility interacting with the particle's field produces parametric $x$-rays. We will also present a theoretical overview of this phenomenon which can be used to generate monochromatic, linearly polarized, directional x-rays. Accelerators with energies ranging from a few $\mathrm{MeV}$ to hundreds of $\mathrm{MeV}$ may be used as drivers for novel parametric $x$-ray generators for various applications requiring the unique properties of these sources.
\end{abstract}

\section{INTRODUCTION}

Parametric x-rays (PX) are a distinct form of radiation produced by a charged particle passing through a crystal structure. The mechanism of PX production can be described as the diffraction of tre virtual photon field of a relativistic particle by the array of atoms in the crystal. The diffracted virtual photons appear as real photons at the Bragg angle corresponding to diffraction of $x$-rays of the observed wavelength by a given crystal plane. This process is illustrated in Fig. 1. The first theoretical treatment of this phenomenon was given by Ter-Mikaelian, 


\section{DISCLAIMER}

Portions of this document may be illegible in electronic image products. Images are produced from the best available original document. 
using a perturbation approach to calculate the electromagnetic fields associated with a charged particle passing through a crystal lattice. Ter-Mikaelian calculated the PX produced in the case of a thin crystal, i.e. Where absorption can be neglected. More complete analyses of PX in thick crystals were done by Barishevsky and Feranchuk ${ }^{2}$ and independently by Garibian and Yang ${ }^{3}$, both in the early $1970^{\prime} \mathrm{s}$.

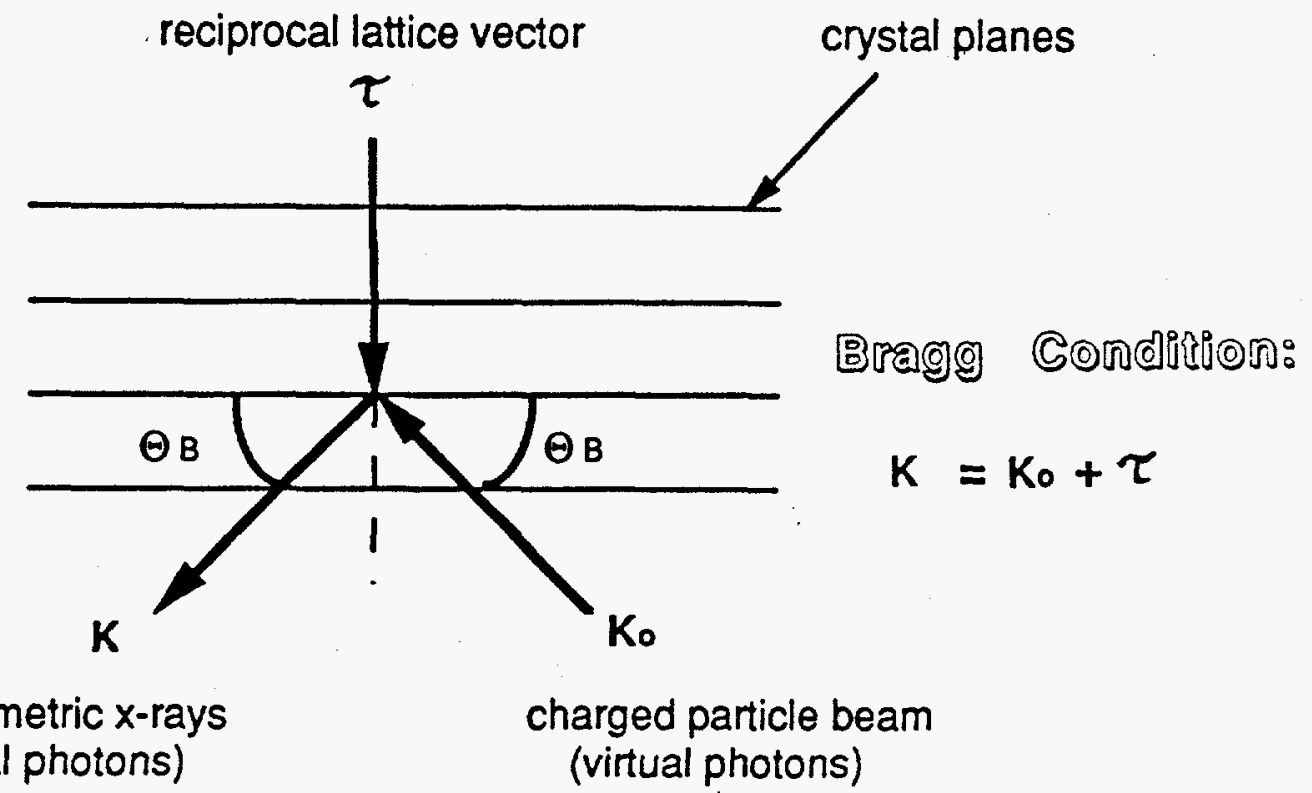

parametric $x$-rays

(virtual photons)

Fig.1. Diffraction of virtual photons associated with a relativistic charged particle by the planes of a crystal.

The first experimental verification of the theory was done by Baryshevsky et at. ${ }^{4}$ in 1985 . These workers produced PX in the $5-25 \mathrm{keV}$ range by passing a $900 \mathrm{MeV}$ electron beam through diamond. subsequently, several experiments were performed to investigate the properties of PX. For example, studies were made of the angular distribution $5.6,7$, the energy dependence in the $200-900 \mathrm{MeV}$ regime ${ }^{6}$ and the spectral distribution at 200-900 $\mathrm{MeV}^{6.8}$ and at 25-MeV $\mathrm{M}^{9}$. In addition, the polarization of $P X$ has been investigated in the case of $900 \mathrm{MeV}$ electrons transmitting a silicon crystal ${ }^{10}$. The experiments cited above were done using primarily diamond and silicon radiators; however the influence on PX production of the $K \alpha$ absorption in Germanium was also investigated by Adishchev et al." PX production by a Gallium Arsenide monocrystal was studied by Afanasenko et al. ${ }^{12}$ The experimental studies have generally confirmed the earlier theoretical predications, while some of the detailed comparisons of angular distributions and bandwidths have not been in complete agreement with theory.

\section{THEORY}

Feranchuk and Ivaskin ${ }^{13}$ have summarized the theoretical expressions for PX and provided detailed numerical examples of PX production by diamondlike, i.e. body centered cubic lattices. The angular distribution of PX intensity can be expressed as 


$$
\begin{gathered}
\frac{\partial^{2} N}{\partial \theta_{x} \partial \theta_{y}}=\sum_{n=1}^{\infty} \frac{e^{2}}{4 \pi} \omega_{B}(n) L_{a}\left[1-\exp \left(-L / L_{B}\right)\right] \frac{\left|g_{\tau}\left(\omega_{B}(n)\right)\right|^{2}}{\sin ^{2} \theta_{B}} \\
\quad x \quad \frac{\left[\theta_{x}^{2} \cos ^{2} 2 \theta_{B}+\theta_{y}^{2}\right]}{\left[\theta_{x}^{2}+\theta_{y}{ }^{2}+\theta_{p h}{ }^{2}\right]},
\end{gathered}
$$

where

$$
\omega_{B}(n)=\frac{n \pi c}{d \sin \theta_{B}} \quad, n=1,2,3 \ldots
$$

is the usual Bragg expression relating the radiation frequency $\omega_{B}$ to the Bragg angle $\theta_{B}$ and the spacing $d$ between crystal planes for a given reflection. The angles $\theta_{x}$ and $\theta_{y}$ are measured relative to the direction in which $x$-rays incident at the Bragg angle would be diffracted by the crystal, with $\theta_{x}$ measured in the plane containing the incident and reflected direction and $\theta_{y}$ perpendicular to this plane. The crystal thickness is $L$ and the $x$-ray absorption length is $L_{a}$. The definition of

$$
\theta_{p h}^{2} \text { is } \theta_{p h}^{2}=\frac{m^{2}}{E^{2}}+\theta_{s}{ }^{2}+\left|g_{0}\right| \text {, }
$$

where $\mathrm{m}$ is the electron rest mass, $\mathrm{E}$ is the incident electron's kinetic energy, and $\theta_{s}{ }^{2}$ represents a mean multiple scattering angle. The Fourier components of the dielectric susceptibility appear as $g_{0}$ and $g_{\tau}$ above, where $g_{0}\left(\omega_{B}\right)$ is the mean value and $g_{\tau}\left(\omega_{B}\right)$ is the value of the Fourier component corresponding to a reciprocal lattice vector $\tau$. This is just the structure factor for the Bragg reflection: $\mathbf{k}=\mathbf{k}_{0}+\tau$, relating the incident wave vector $k_{0}$ to the reflected wave vector $k$ by addition of the reciprocal lattice $\boldsymbol{r}$, which is/ gerpendicular to the crystal planes causing the reflection, where $|\tau|=2 \pi / \mathrm{d}$ (see Figure 1 ). For an incident virtual photon, we also have the relation $k_{0} \cdot v=\omega_{B}$.

The appearance of $\cos ^{2} 2 \theta_{B}$ in eq. (1) indicates that the relative components of the intensity in the $\theta_{x}$ and $\theta_{y}$ directions can vary with $\theta_{B}$. These components consist of electric vectors polarized in the $x$ and $y$ directions. For example, for $\theta_{B}=45^{\circ}$, only the $y$ component of polarization would be non-zero. The angular width of Eq. (1) is

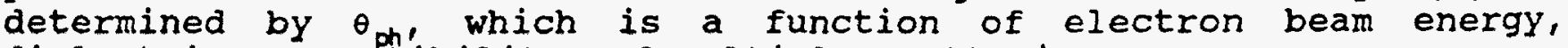
dielectric susceptibility and multiple scattering.

Fig. 2 a illustrates a contour plot of the angular distribution of intensity in Eq.(1) for the case of $\theta_{B}=22.5^{\circ}$. The angles $\theta_{x}$ and $\theta_{y}$ are in units of 


$$
\gamma_{\text {eff }}^{-1}=\left[\frac{m^{2}}{E^{2}}+\left|g_{0}\right|\right]^{3 / 2} \text {. }
$$

Note that the peak intensities occur at $\theta_{x}=0, \theta_{y}^{-} \pm 1$ in these units. The intensity contours are in arbitrary units, with the peak contour value of 0.25 and contour steps of 0.017 in Fig.2a and steps of 0.018 in Fig. 2b. In Fig. 2b we show the angular distribution from Eq. (1) plotted for the case of $\theta_{B}=45^{\circ}$, in which the radiation is polarized in the $\mathrm{y}$-direction, assuming an electron beam without divergence.
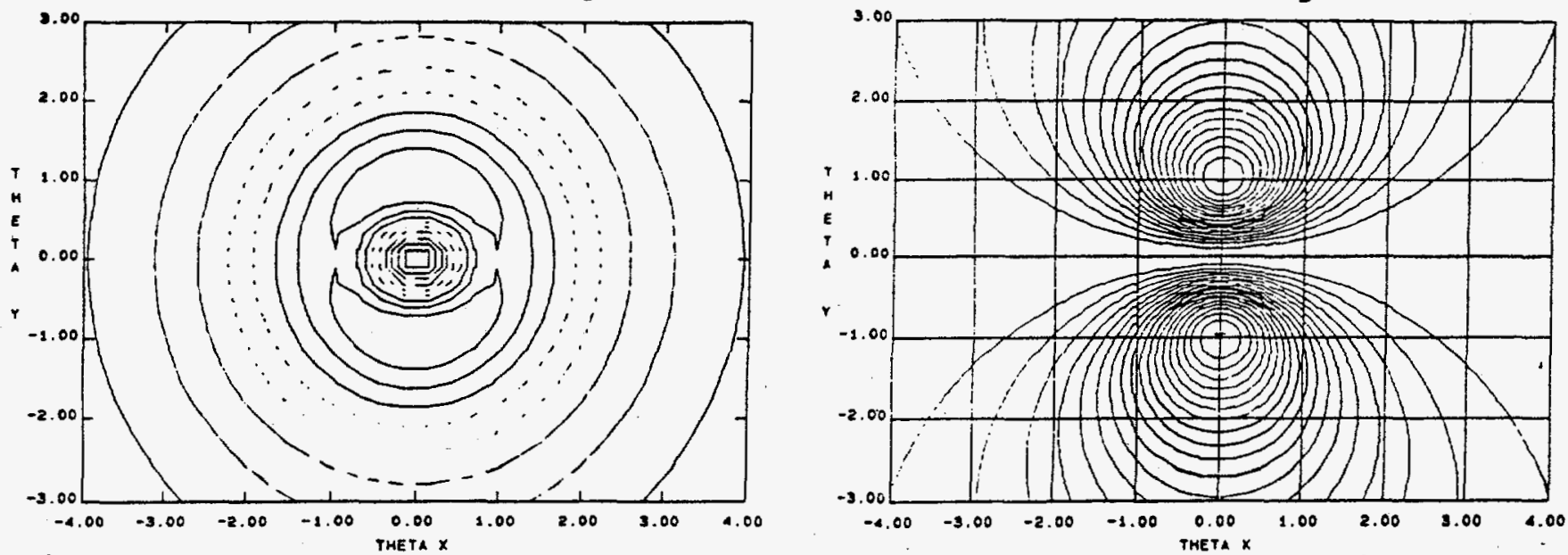

Fig. 2a. Contour plot of angular distribution of intensity in Eq. (1) for $\theta_{B}=22.5^{\circ}$. The direction $\theta_{x}=\theta_{y}=0$ corresponds to the direction of $R$ in Fig. 1. Fig. 2b. The case when $\theta_{B}=45^{\circ}$ and the radiation is polarized with the electric vector along the $\theta_{y}$ direction.

The frequency distribution of $\mathrm{PX}$ has a narrow bandwidth and the distribution is given by

$$
\frac{\partial N}{\partial U}=N_{1} J_{1}(U) \text {, }
$$

where

$$
J_{1}(U)=\frac{1+U^{2}\left(1+\cos ^{2} 2 \theta_{B}\right)}{\left[1+U^{2}\right]^{3 / 2}},
$$

with $U=\frac{\sin \theta_{B}}{\cos \theta_{B}} \frac{\left(\omega-\omega_{B}\right)}{\omega_{B} \theta_{p h}}$.

The factor $N_{1}=(\pi / 2) N_{0}$, where $N_{0}$ is defined to be all the factors in Eq. (1) which are independent of $\theta_{x}$ and $\theta_{y}$, i.e. everything but the last term in Eq. (1) for a given value of $\mathrm{n}$ in the sum. From Eq. (6) it can be seen that the line width will depend on $\theta_{B}$ and $\omega_{B}$ as well as on the electron beam energy, the multiple scattering, and the mean dielectric susceptibility through $\theta_{\text {ph. }}$ Eq. $(3)$. These dependencies have not been 
fully investigated. As yet, there is no satisfactory treatment of multiple scattering for $\mathrm{PX}$ and its effects on linewidth.

The total number of photons detected in a solid angle $\theta_{D}^{2}$ can be obtained by integrating Eq. (1) over $\theta_{0}^{2}=\theta_{x}{ }^{2}+\theta_{y}^{2}$. This yields

$N_{D}=N_{1}\left(1+\cos ^{2} 2 \theta_{B}\right)\left\{\ln \left[\frac{\theta_{D}^{2}+\theta_{p h}^{2}}{\theta_{p h}{ }^{2}}\right]-\frac{\theta_{D}^{2}}{\theta_{D}{ }^{2}+\theta_{p h}{ }^{2}}\right\}$.

Since the angular distribution of PX given by Eq. (1) falls off slowly with angle, the integrated intensity $\mathrm{N}_{0}$ above is seen to depend on the detector size even when $\theta_{D}>>\theta_{p h}$.

The above discussion highlights some of the unique properties of PX as a radiation source: it is a narrow band, tuneable, polarizable, and can be directed out of the electron beam direction by Bragg reflection. Baryshevsky and Feranchuk ${ }^{14}$ have performed a comparative analysis of $P X$ with other sources of $x$-rays such as synchrotron, bremsstrahlung, cerenkov, transition radiation, and channeling radiation. According to these authors, $\mathrm{PX}$ is the most effective source of quasimonochromatic focused $x$-rays. For example, for $10 \mathrm{keV} x$-rays with a bandwidth $\Delta \omega / \omega=$ $10^{-3}$, they claim that $\mathrm{PX}$ produced by a $50 \mathrm{MeV}$ beam has a flux comparable to that produced by a $3 \mathrm{GeV}$ synchrotron. Recent work by Caticha ${ }^{15}$, based on the dynamical theory of x-rays, has elucidated the role of two distinct PX modes: the quasi-Cerenkov mode discussed above which gives a very narrow, intense peak, and a "transition diffracted radiation" mode which is the counterpart of the usual Bragg reflection of $x$-rays, which gives a broader spectrum resembling the Darwin peak. These modes have not yet been distinguished experimentally.

\section{EXPERIMENTAL PX STUDIES IN SILICON USING A 90 MEV ELECTRON BEAM}

\subsection{Experimental setup and technique}

In January of this year, we performed an experiment to observe parametrically generated $x$-rays (PX) produced by the interaction of a 90 Mev electron beam with a silicon crystal, using the linear accelerator at the Naval Postgraduate school in Monterey, California.

A schematic of our PX experimental setup is shown in Fig. 3 . The figure shows a silicon crystal oriented for production of PX from the <011> planes. The electron beam is incident on these planes at a Bragg angle of 22.5 degrees. The detection angle relative to the electron beam direction is 45 degrees. In order to successfully generate PX at a given Bragg angle, the direction of the electron beam relative to the diffracting planes must be maintained within a tolerance of a few milliradians. In order to accomplish this, the experimental arrangement makes novel use of optical transition radiation (OTR) diagnostics (refs. 16-20) to monitor the electron beam and its direction with respect to the crystal planes. This greatly simplifies the experiment in comparison to previous studies (refs. 4-12) which relied on the 
monitoring of channeling radiation (CR) to align the crystal. To observe $C R$, which is generated in the forward direction, i.e. nearly parallel to the electron beam velocity vector, the beam had to be bent out of the line of sight in those experiences. As the diagram indicates, both the OTR and $P X$ are observed at large angles relative to the beam direction, and hence beam deflection is unnecessary.

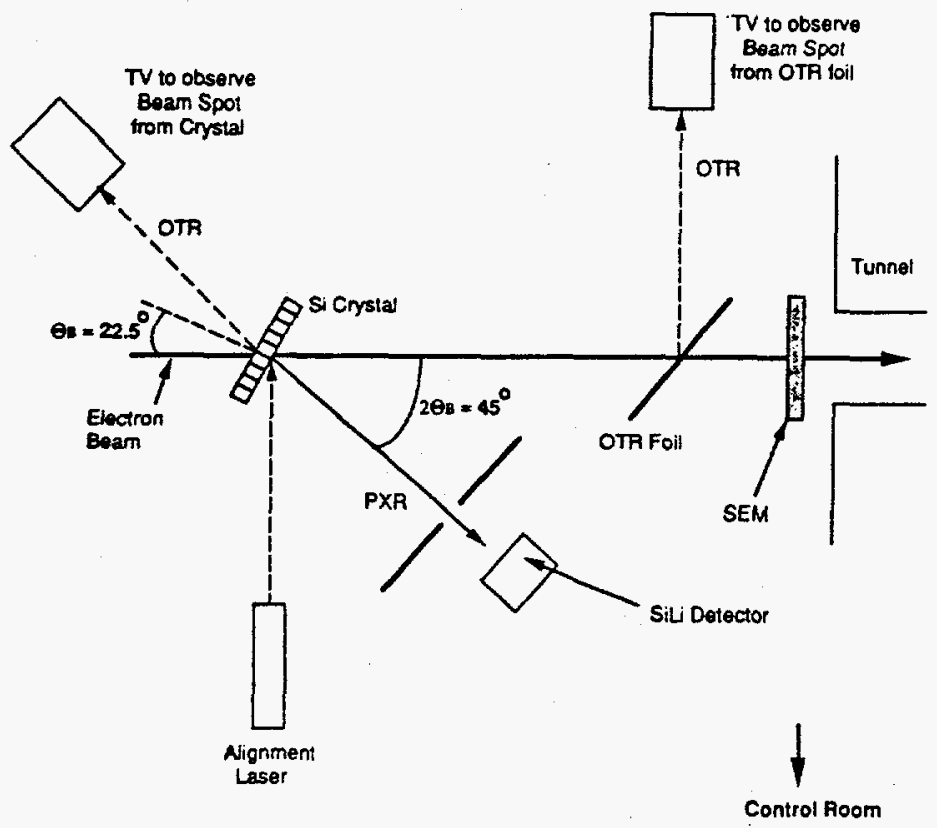

Fig 3. Apparatus for NPS parametric $x$-ray experiment. The electron beam enters from the left, striking the silicon crystal. The $x$-rays emerge at the Bragg angle and are detected by a sili detector. The electrons continue on to an OTR fnil monitor station and an SEM.

a)

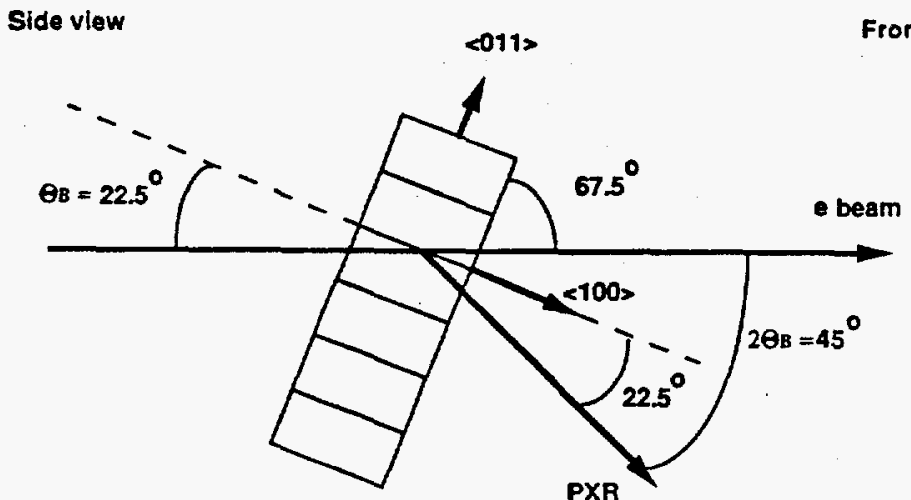

b)

ront View (along beam direction)

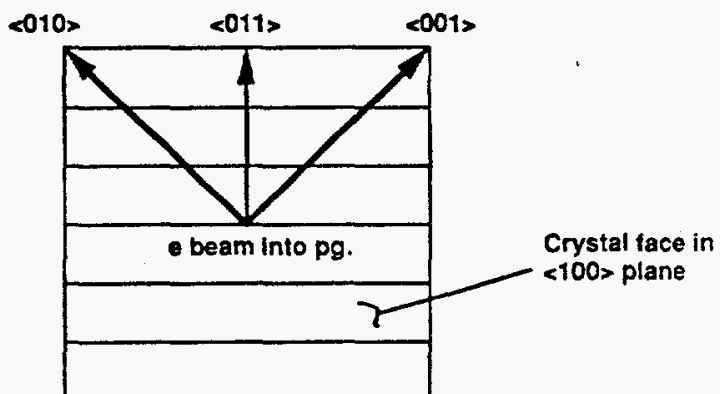

Fig. 4a. Detailed view of crystal orientation relative to the electron beam for PX generation from the <011> planes of silicon at a Bragg angle of $22.5^{\circ}$. Fig. $4 \mathrm{~b}$. Front view of silicon crystal showing the boundaries of the area of the crystal that was etched to $20 \mu \mathrm{m}$ thickness. 
A detailed drawing showing the silicon crystal's orientation is given in Fig. $4 a$. The crystal is cut so that the <011> planes are oriented at right angles with respect to the crystal face, whose normal is the <100> direction. A square $4 \mathrm{sq} . \mathrm{cm}$. area centered on the beam axis is etched out of the crystal to reduce its thickness to 20 microns. Fig. $4 \mathrm{~b}$ shows the boundaries of the etch with respect to the crystal lattice vectors. The side of the crystal away from the electron beam is an optically mirrored surface. The etched surface (nearest the electron beam) is also highly reflective in the visible. The crystal is initially oriented so that the <011> is aligned to the vertical (normal to the plane of incidence). A theodolite is used to position the <011> axis to within a milliradian of the vertical, as defined by a plumbline. The crystal is oriented with the help of a two-axis goniometer. The Bragg angle is controlled by a stepper with submilliradian accuracy, and the rotation angle out of the plane is stepper controlled to within 0.5 milliradians.

To obtain energy spectra of the PX generated in the crystal, a liquid nitrogen cooled sili detector was used in conjunction with an ortec Corporation pulse height electronics and a Nucleus Inc. PC multichannel analyzer. The sili detector is positioned one meter away from the crystal's vertical rotation axis. To minimize absorption of the PX in air, a vacuum line extends to within a few centimeters of the detector's Be input window. A one mil Kapton window, $2 \mathrm{~cm}$. in diameter, allows the $x$-rays to exit the vacuum chamber. The sili detector is aligned with the help of a HeNe laser which is reflected by the mirrored back surface of the silicon crystal.

The laser is also used to define an optical line of sight for the alignment of two low light level television cameras and associated optical components. The cameras used were a Hamamatsu Model C-1000 SIT and a Cohu Model $4815 \mathrm{CCD}$. These cameras ar'z used to observe the beam via optical transition radiation, which is generated when the electron beam intercepts the crystal or a foil placed downstream from the crystal and oriented at 45 degrees relative to the electron beam velocity vector. The cameras are focused at the crystal or a foil, respectively, to provide a means to dynamically monitor the electron beam at these two axial positions which define the path of the electron beam. The beam is allowed to drift freely between these two points. An alignment process to insure coincidence of the laser defined line of sight and the electron beam trajectory is performed iteratively.

\subsection{Measurements of the PX spectra}

Pulse height analysis is performed to obtain the energy spectrum of the $P X$ radiation detected by the sili detector. In order to accomplish this, the electron beam current must be reduced so as not to saturate the detector and allow pulse counting below the maximum counting rate $(10,000 \mathrm{cts} / \mathrm{sec})$. The electron beam linac produces a $100 \mathrm{microsecond}$ pulse at a repetition rate of $60 \mathrm{~Hz}$. It was found to be necessary to reduce the electron beam current to about a nanoampere average current to allow the pulse height analysis (PHA). To accomplish this the linac was first tuned at a current of about $0.1 \mu$. The grid of the injector gun was then turned off to achieve the nanoampere operating current. 
It was observed that, with proper tunings, the position of the beam monitored with the help of OTR did not change and remained stable for periods up to an hour as the electron beam current was reduced. This was very important since at the very low current required for PHA, it was not possible to monitor the beam position directly, because the OTR produced was too low to be detected by either camera. A second spectrum was obtained for PX generation from the <111> planes, by orienting the crystal as shown in Figure 5. The Bragg angle and detector angle of 22.5 and 45 degrees respectively were maintained by rotating the crystal to an angle of 102.76 degrees from the electron beam direction, as shown.

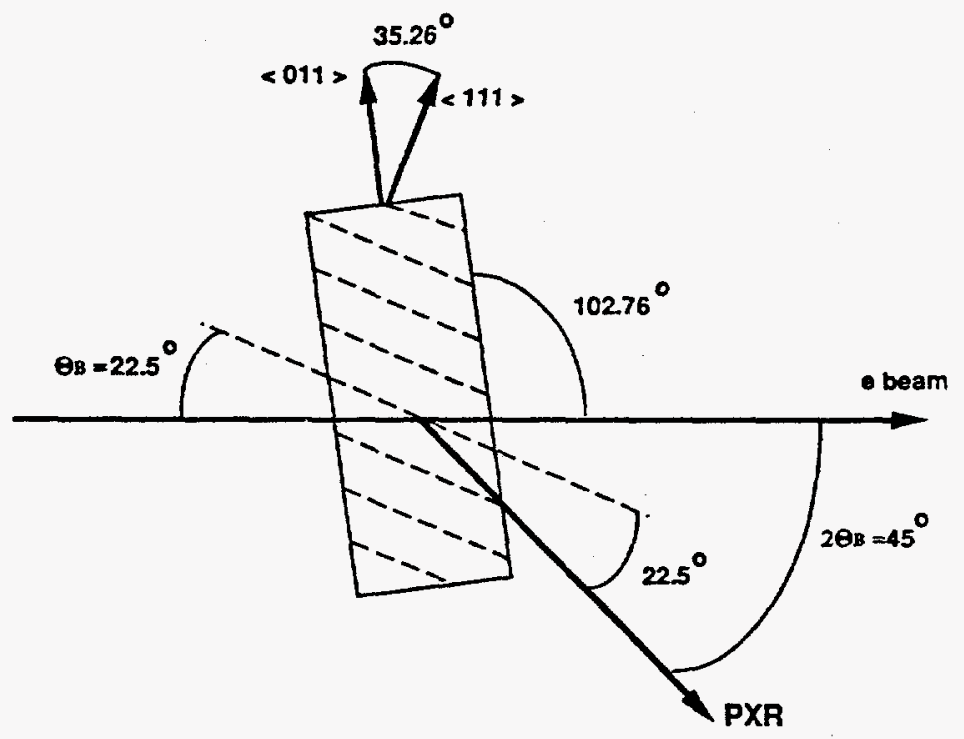

Fig. 5. Orientation of crystal for PX generation from <111> planes of silicon.

\subsection{Imaging polarized parametric $x$-rays}

An image of the $\mathrm{PX}$ radiation pattern was made for a Bragg angle equal to 45 degrees. The arrangement for this experiment is shown in Fig. 6 . To image the PX pattern, a $2.54 \mathrm{~cm}$ Hamamatsu liner diode array was employed. Each detector of the array consists of a 50 micron wide by $2.5 \mathrm{~mm}$ high photosensitive element. The array was mechanically scanned over a $17 \mathrm{~mm}$ aperture to obtain a two dimensional profile of the $x$-ray pattern at 0.5 meters from the crystal source. The angular aperture was ample to image the PX pattern whose full width is estimated to be 20 milliradians. The array housing and drive assembly are physically attached to the vacuum port at 90 degrees relative to the beam axis, as shown in the figure. The array is exposed to the vacuum of the accelerator beamline. To center the array, which is also sensitive to visible light, a second laser is used to produce a beam coincident with the laser beam previously used for the experimental setup shown in

Fig. 3. The second laser is used in conjunction with a beamsplitter as shown in Fig. 6. The imaging experiment was performed at a current of 0.1 microamperes. 200 pulses were integrated at each position of the array. 


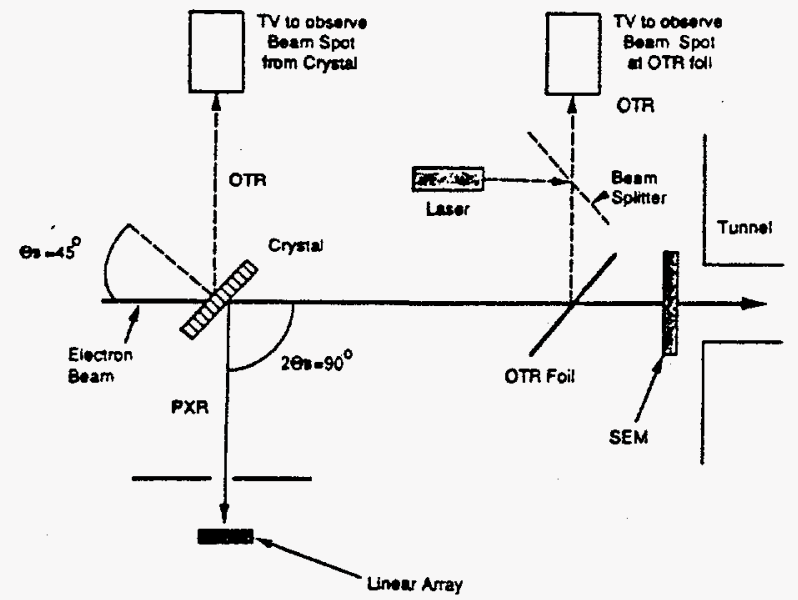

Fig. 6. Experimental arrangement for imaging polarized PX production at a Bragg angle of $45^{\circ}$ relative to the <011> planes of silicon. The detector was a linear diode array.

\section{RESULTS}

Spectra taken at the bragg angles of 22.5 degrees for PX generation from the <011>, and <111> planes are shown in Figs. $7 a$ and $7 \mathrm{~b}$ respectively. Note that the $n=2$ mode is the first mode allowed for $P X$ generation from the <011> plane. The Bragg condition predicts the energies for an incidence angle of 22.5 degrees are $8.42 \mathrm{kev}$ and 5.16 kev respectively for the <011> and <111> planes.
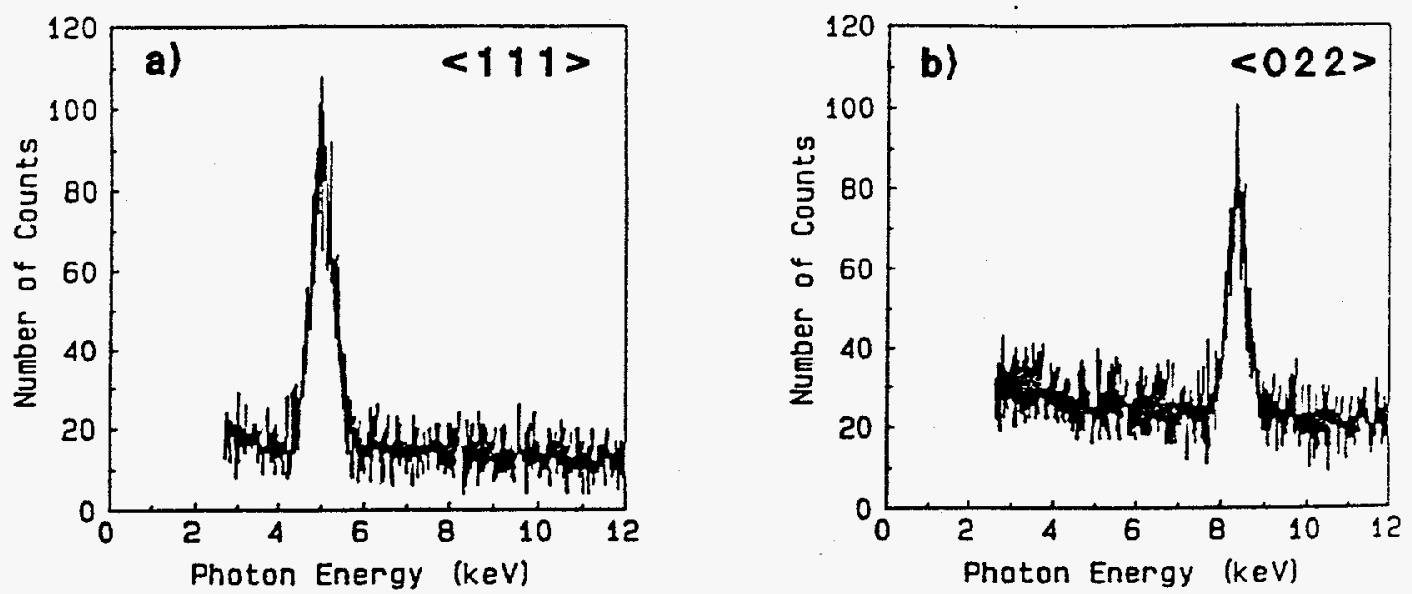

Fig. 7a. Measured spectrum of $x$-rays from the <111> reflection of silicon at Bragg angle of $22.5^{\circ}$, with peak at $4.96 \mathrm{keV}$. Fig. $7 \mathrm{~b}$. The spectrum from the $\langle 022\rangle$ reflection at Bragg angle $22.5^{\circ}$, with peak at $8.44 \mathrm{keV}$.

Table 1 contains the measured energies and line widths, i.e. full widths at half the maximum intensity (fwhm) for the two reflections, as well as the theoretical values from Equations (2) and (4). The agreement is excellent except for the measured <022> line width which is narrower than predicted. This may be a result of the effect of the noise level on determining the experimental peak height. 
TABLE 1

COMPARISON OF EXPER. F THEORETICAL SPECTRAL OUANTITIES IN UNITS OF EEV

$\begin{array}{lllllr}\begin{array}{l}\text { reflect. } \\ \text { plane }\end{array} & \begin{array}{l}\text { Bragg } \\ \text { angle }\end{array} & \begin{array}{c}\omega_{B} \\ (\mathrm{exp})\end{array} & \begin{array}{l}\omega_{B} \\ (\text { thy) }\end{array} & \begin{array}{l}\text { Fwhm } \\ (\exp )\end{array} & \begin{array}{l}\text { Fwhm } \\ \text { (thy) }\end{array} \\ <022\rangle & 22.5^{\circ} & 8.44 & 8.42 & 0.44 & 0.73 \\ <111\rangle & 22.5^{\circ} & 4.96 & 5.16 & 0.56 & 0.56\end{array}$

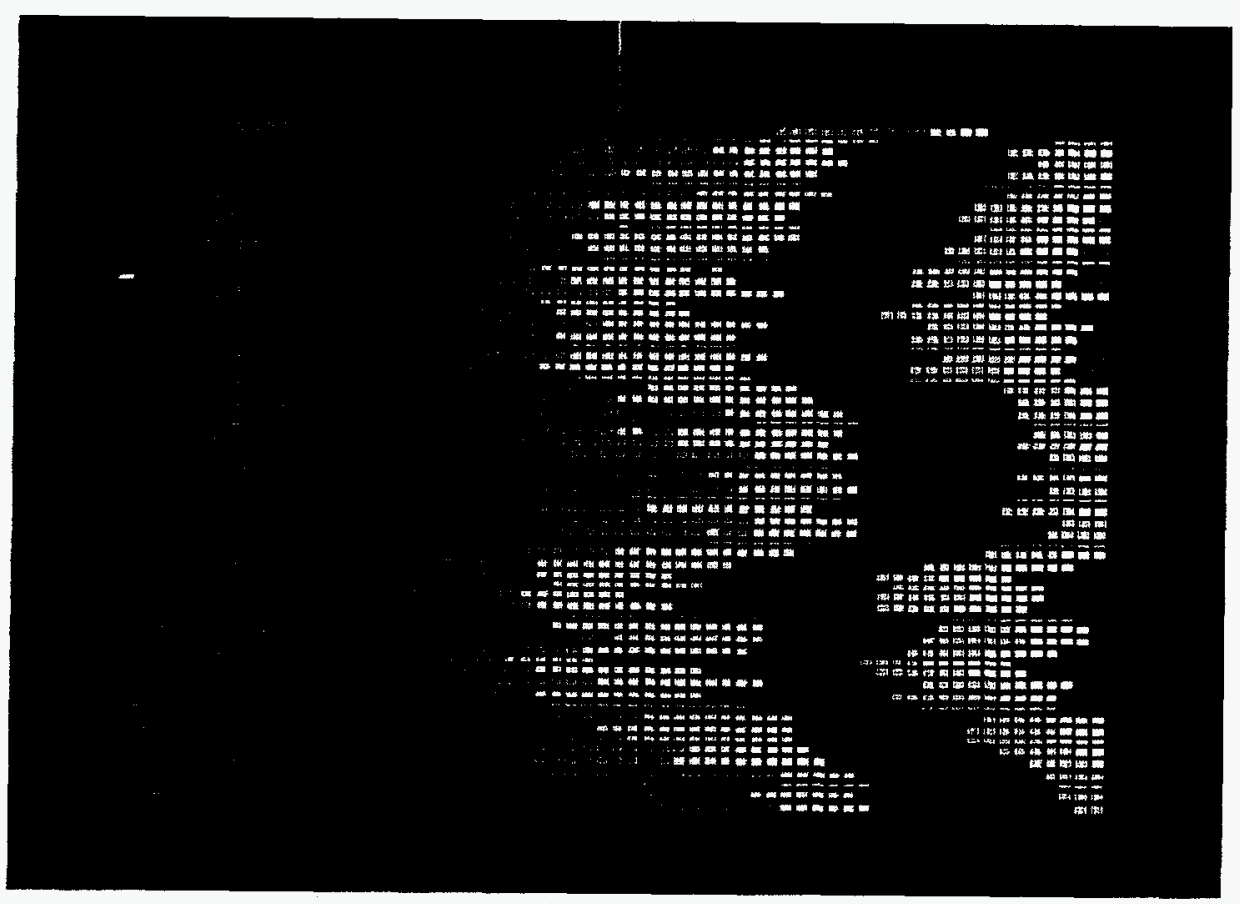

Fig. 8. Color coded distribution of PX intensity for the case of a reflection from the <011> planes at $\theta_{B}=45^{\circ}, \omega_{B}=4.55 \mathrm{keV}$. The peaks occur on the right hand edge of the picture, which corresponds to $\theta_{x}=0$ in Fig. $2 \mathrm{~b}$.

The two dimensional PX pattern is shown in Fig. 8. The figure shows a contour map of intensities which was color coded, with the peak intensities in red. The peak intensities corresponding approximately to $\theta_{x}=0, \theta_{y} \pm 1$ in Fig. $2 b$ appear along the right edge of this image. The theory predicts a vertically polarized intensity pattern. Unfortunately a quantitative comparison is not possible because of the very low number of counts in this data. Also, the pattern is not centered on the array. Before higher quality data could be obtained, the silicon crystal became mechanically distorted and further PX data was judged to be unreliable. Nevertheless, there is evidence in this figure that the predicted vertically polarized pattern has been observed. We believe that this is the first clear image of the angular distribution pattern of polarized PX to be published. This experiment did not include absolute measurements of intensities; Eq. (7) predicts, for $\theta_{0}=2 \gamma_{\text {eff }}^{-1}$, that $N_{D}=10^{6}-10^{7}$ photons $/\left(\mathrm{sec}-\mathrm{cm}^{2}\right)$ for the case of $\theta_{B}=45^{\circ}$. In future work we 
will concentrate on obtaining quantitative images of the PX pattern and absolute intensity measurements.

In conclusion, we have demonstrated that the observed radiation possessed the energy, linewidth, and angular pattern predicted by the theory of parametric $x$-ray generation. Further more, this radiation was produced with a linac of modest beam quality, and moderate energy, i.e. a divergence of about I milliradian and an energy of $90 \mathrm{MeV}$.

\section{REFERENCE8}

1. M. L. Ter-Mikaelian, High Energy Electromagnetic Processes in Condensed Media, New York, Wiley-Interscience, 1972, pp. 332-335.

2. G. Baryshevsky and I. D. Feranchuk, "Transition Radiation of Gamma Rays in a Crystal," Sov. Phys. - JETP 34, 502-504, 1972.

3. G. M. Garibyan and C. Yang, "Lateral spots of X-ray Transition Radiation in Crystals and their Effect on the central spot, "Sov. Phys. - JETP, 34, 495, 1972; 36; 631, 1973.

4. V. G. Baryshevsky et al., "Experimental observation of the Parametric X-rays from Ultrarelativistic Electrons," J. Phys.D 19, $171-176,1986$.

5. V. G. Baryshevsky et al., "Angular Distribution of Parametric Xrays," Phys. Lett. 110A, 477-479, 1985.

6. Yu. N. Adishchev et al., "Measurements of Parametric X-rays from Relativistic Electrons in Silicon Crystals, "Nucl. Instr. and Methods A248, pp. 49-55, 1987.

7. Yu. N. Adishchev et al., "Angular Distribution of Parametric (QuasiCherenkov) Radiation," Sov. Phys.-JETP, 66, pp. 1107-1111, 1987.

8. Yu. N. Adishchev et al., "Spectral Distribution of Parametric X-rays from Ultrarelativistic Electrons in a Sov. Phys. Tech. Phys. 33, pp. $461-465,1988$.

9. A. V. Shchagin et al., "A Fine structure of Parametric X-ray Radiation from Relativistic Electrons is a Crystal," Phys. Lett. A 148 , pp. 485-488, 1990 .

10. Yu. N. Adishchev et al, "Measurement of Spectral and Polarization Characteristics of Parametric X-rays in a Si Crystal," Nucl. Instr. and Methods in Phys. Res. B44, pp. 130-136, 1989.

11. Yu. N. Adishchev et al., "Influence of Ka Absorption in (111) Ge Crystal on spectral Yields of Parametric X-rays," Phys. Lett. A 147, pp. 326-328, 1990 .

12. V. P. Afanasenko et al., "Detection of Parametric X-ray Radiation of a Ga As Monocrystal," Phys. Lett. A, 141, pp. 311-313, 1989.

13. I. D. Feranchuk and A. V. Ivashin, "Theoretical Investigation of the Parametric X-ray Features," J. Physique 46, pp. 1981-1986, 1985.

14. V. G. Baryshevsky and I. D. Feranchuk, "A Comparative Analysis of Various Mechanisms for the Generation of X-rays by Relativistic Particles," Nucl. Instrum. and Methods in Phys. Res. 228, pp. 490495,1985 .

15. Ariel Caticha, "Transition-diffracted Radiation and the Cerenkov Emission of X-rays," Phys. Rev. A, vol. 40, No. 8, pp. 4322-4329, 1989 . 
16. R. Fiorito et al. "Transition Radiation Diagnostics for Intense Charged Particle Beams, Proc. of the 6th Intl. Conf. on Intense High Power Particle Beams, Kobe, Japan (June 1986) (ed. C. Yamanaka, Osaka University, Osaka, Japan).

17. S. Iversen et. al. "Charged Particle Divergence Measurements Using Optical Transition Radiation," Proc. of Particle Accelerator Conference, Washington, DC, (March 1987).

18. D. Rule, "Transition Radiation Diagnostics for Intense Charged Particle Beams," Nucl. Instrum. and Methods B24/25, p. 901, 1987.

19. A. Lumpkin, et al. "Initial OTR Measurements of the Electron Beam for the Boeing FEL Experiment, Nucl. Instrum. and Methods A296, p. $150,1990$.

20. D. Rule, et. al. "Comparative Analysis of OTR Based Electron Beam Emittance Measurements for the Los Alamos FEL, Nucl. Instrum. and Methods A296, pp. 739-743, 1990.

\section{DISCLAIMER}

This report was prepared as an account of work sponsored by an agency of the United States Government. Neither the United States Government nor any agency thereof, nor any of their employees, makes any warranty, express or implied, or assumes any legal liability or responsibility for the accuracy, completeness, or usefulness of any information, apparatus, product, or process disclosed, or represents that its use would not infringe privately owned rights. Reference herein to any specific commercial product, process, or service by trade name, trademark, manufacturer, or otherwise does not necessarily constitute or imply its endorsement, recommendation, or favoring by the United States Government or any agency thereof. The views and opinions of authors expressed herein do not necessarily state or reflect those of the United States Government or any agency thereof. 